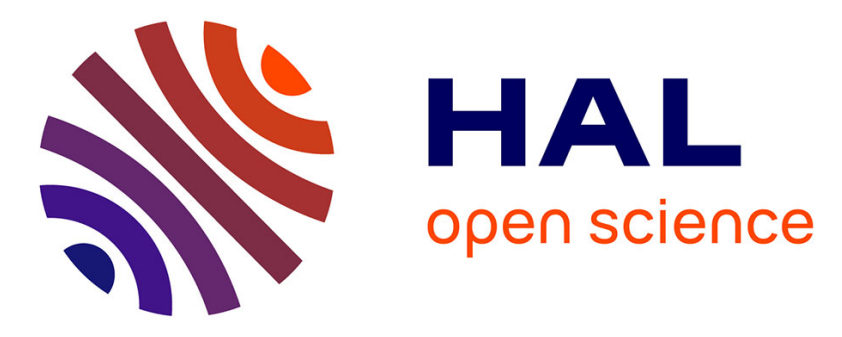

\title{
Modeling and analysing ubiquitous systems
}

Amara Touil, Jean Vareille, Lherminier Fred, Philippe Le Parc

\section{To cite this version:}

Amara Touil, Jean Vareille, Lherminier Fred, Philippe Le Parc. Modeling and analysing ubiquitous systems. 15th IEEE International Conference on Emerging Techonologies and Factory Automation (ETFA2010), Sep 2010, Bilbao, Spain. pp.MF-004529. hal-00526699v2

\section{HAL Id: hal-00526699 \\ https://hal.univ-brest.fr/hal-00526699v2}

Submitted on 15 Oct 2010

HAL is a multi-disciplinary open access archive for the deposit and dissemination of scientific research documents, whether they are published or not. The documents may come from teaching and research institutions in France or abroad, or from public or private research centers.
L'archive ouverte pluridisciplinaire HAL, est destinée au dépôt et à la diffusion de documents scientifiques de niveau recherche, publiés ou non, émanant des établissements d'enseignement et de recherche français ou étrangers, des laboratoires publics ou privés. 


\title{
Modeling and analysing ubiquitous systems
}

\author{
Amara Touil ${ }^{*}$, Jean Vareille*, Fred Lherminier ${ }^{\dagger}$, and Philippe Le Parc* \\ *Université Européenne de Bretagne, France \\ Université de Brest - EA3883 LISyC \\ Laboratoire d'Informatique des Systèmes Complexes \\ 20 av. Victor Le Gorgeu, BP 809, F-29285 Brest \\ Email: philippe.le-parc@univ-brest.fr \\ †Terra Nova Energy \\ Z.I. de Kergaradec, 28 rue Victor Grignard, F-29490 Guipavas
}

\begin{abstract}
The growth of industrial activities during the last decades and the diversity of industrial products require standards and common methodologies for building and integrating different parts. It is also required that working groups use the same terminologies and concepts needed for each domain. The Model Driven Engineering (MDE) approach aims to give an answer while using a high level method based on models and transformations.

In this paper, we use the MDE approach to model ubiquitous systems. Those systems are composed of devices interconnected through various kinds of network and offer to get and send information. We present a model for this class of system and its use in the field of energy while studying real cases from our industrial partner Terra Nova Energy (TNE).
\end{abstract}

Keywords: Model Driven Engineering, Analysis, Telecontrol Systems.

\section{INTRODUCTION}

Ubiquity is often define as being able to be in several places at the same time. In the field of Information Technologies, this definition can be refined in, at least, two ways that may be apparently opposites. The first approach is to considered as in [1] that users are surrounded with "intelligent" systems, that may delivers them needed information: in this case, computing facilities are used to locate users, to understand their environment, to anticipate their needs and to make it possible that all information they require is available anywherere at anytime. The second approach, is to offer people to be able to get information from a system located somewhere and to be able to act safely on it: in this case computing facilities are used to make it possible to be "virtually" present in several places at the same time. We place our work in the second approach in order to model and analyze telecontrol application as a kind of ubiquitous systems.

Terra Nova Energy (TNE) is an innovative company that provides solutions for data mining in the fields of electricity, hydraulic and pulse-energy. It integrates sensing, acting and communicating devices in telecontrol systems, for collecting data from different sites using various technologies. In order to improve its development, this company is facing two problems: how to conceive their systems as automatically as possible and how to fasten their on-site deployment?

To answer these questions, we propose to use a model based approach, relying on specific components for telecontrol domain, and libraries to integrate industrial parts coming from various providers. Our idea is to build a framework [2] following the Model Driven Engineering (MDE) methodology [3][4] to create a telecontrol ontology and proper transformations for building, generating, analyzing and deploying such ubiquitous telecontrol systems.

This paper is organized as follows: first of all, the Model Driven Engineering approach and transformations that may be conducted are briefly introduced. Second, we introduce the generic meta-model proposal. Third, this general meta-model is applied to our case study, while defining an instance for TNE. Then we explain how to carry a static analyzis on a given instance and show first results. Finally we discuss our method and we finish by further work.

\section{RELATED WORKS}

In this section, we introduce some notions about the MDE approach and how to conduct transformations on models.

A model is an abstracted view of a system that expresses related knowledge and information. It is defined by a set of rules, used to interpret the meaning of its components[5]. A model is defined according to a modeling language that can give a formal or a semi formal meaning description of a system depending on modeler's intention.

The model paradigm has gained in importance in the field of systems engineering since the nineties. Its breakthrough was favoured by working groups like the Object Management Group (OMG)[6] that has normalized modeling languages such as Unified Modeling Language (UML). This group also provides the Model-driven architecture (MDA) software design standard. The MDA is the main initiative for Model Driven Engineering (MDE) approach.

According to OMG, four abstraction levels have to be considered: a meta-meta-model that represents the modeling language and which is able to describe itself; a meta-model level that represents an abstraction of a domain and which is defined through the meta-meta-model; a model level that gives an abstraction of a system as an instance of the meta-model; finally, the last abstraction level is the concrete system.

In order to breath life in models [7], model transformations aims to exceed the contemplative model view to a more productive approach, towards code generation, analysis and test, simulation, etc. Models are transformed into other models 
that may be manipulated by specific tools or that may be transformed again into other models. A transformation may be done in order to conduct analysis.

Static analysis deals with observing system and checking some properties before real system creation, production or code implementation. As example, formal verification and model checking techniques [8][9] are used for verifying models. Behavioural analysis are not beyond in the scope of this article. Static analysis are only conducted in simple cases, but relevant ones for TNE. In this paper we will focus on placement but others studies have been conducted (cost, bandwith,...). As soon as the model is created, it may give answers to the engineer: Are my sensors, repeaters, routers placed in a proper location? Are there better configuration? etc.

Next section describes the meta-model proposed for telecontrol and its derivation for TNE's remote monitoring needs.

\section{PROPOSED META-MODEL}

In this section, we, describe the meta-model we are proposing to specify systems based on communicating objects. Only a high level view and the main concepts are presented.

\section{A. Generic meta-model}

At a first abstraction level, we built our domain around systems containing entities that communicate with each other as stated in [10]: "A system is a construct or collection of different elements that together produce results not obtainable by the elements alone". In the meta-model proposal (Figure 1), a system, modeled by System, can contain other systems according to the ownedSystem reference, in order to provide the "system of the system" notion. Regarding entities, they are modeled by Entity and its system containment is referenced by the itsEntity relationship. Entity paradigm in our meta-model aims to be a generic and general concept formed by extracting common features from our specific telecontrol domain.

Moreover, entities are described using several related concepts trying to keep information about their physical properties, communication facilities or behaviour :

- Structure element defines the interrelation or arrangement of different physical parts or the organization of elements that provides coherence, shape and rigidity to an entity. It may describe mechanical, chemical or biological aspects.

- Actions performed by entities are described by the Task concept. They can be internal, such as making computation, external such as sending information and also connected to the real world such as sensing or acting on a real device.

- ContactInterface element allows connection between entities. It can be a physical contact, or a logical interface depending on the specification level, on the refinement degree or on its own structure.

- An entity may contain Data related to itself or to its environment.

- Message element provides data exchange between entities. To send (or to receive a message) from entity
A to entity B, A and B must be connected through ContactInterfaces, directly or, it may exist a path of entities that may forward messages.

- A Behavior concept is added to each entity, in order to describe the different tasks it may realize. From this concept, code generation or simulation may be refined.

As the Entity concept is very general, in order to improve our generic meta-model, different sub-entities have been specialized using the concept of heritage:

1) Device represents a physical component such as a sensor, a router, a computing unit, etc.

2) Medium element represents an entity deployed and used between other entities to specify communication's medium. In classical approaches, medium is often ommitted and considers as perfect links. In our work, medium has its own structure, behavior, contact interfaces, datas, messages and tasks. Different mediums may be described and classified following their own specifications, wired or wireless for example.

3) User element is used to model a person interacting with other entities. Like other entities User can have different presentation depending on model view.

4) The different entities composing the system are under some physical conditions and constraints that affect and influence the global behaviour. In our meta-model we introduce the Environment concept to describe such conditions. . Environment acts on internal elements in order to build a global circumstances of evolving system and gives context for ubiquitous entities and systems that are context-aware.

5) The concept of time is crucial for telecontrol systems that is why a TimeClock entity is added to our generic meta-model for measuring, recording or indicating time.

The presented generic meta-model intends to specify any kind of systems containing communicating objects. At this abstraction level, we present just a first facet (named Basic package), without getting into all the details of the metamodel.

\section{B. Terra Nova Energy meta-model}

According to the concept of inheritance, the Terra Nova Energy meta-model is built as an extention and a generalization of the generic meta-model. The first element is the TNESystem that inherits from the generic element System. Other elements have been spread over two sets: generic devices and off the shelf devices that could be grouped in a specialized library.

1) Terra Nova Energy's generic devices: This first set of elements represents generic devices that allow to add a new industrial component to a library or to build a specific system.

- BoxaNova models the main device of TNE systems. It collects information, sends orders and manages the entire flow of data between different devices and users. A BoxaNova device is generally created by some others devices depending on system configuration and deployment.

- Router models a device that handles message transfers between different TNE elements. It handles also some 


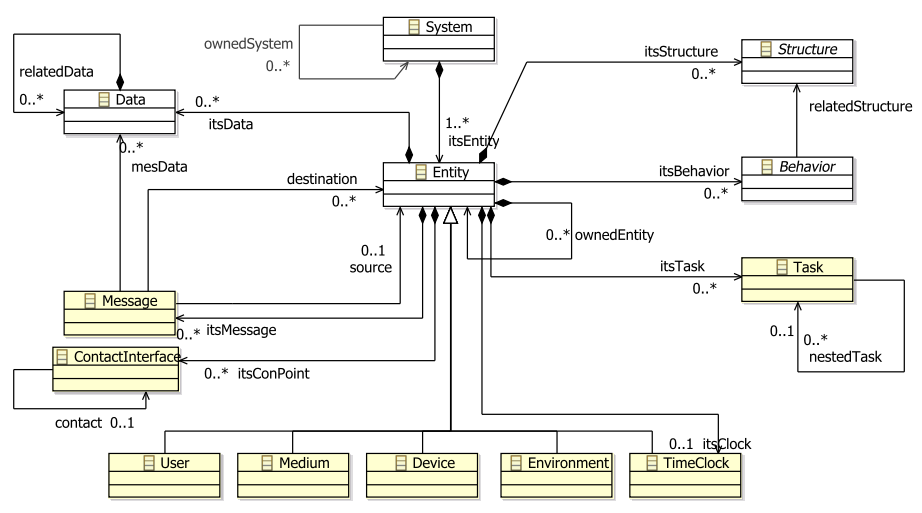

Fig. 1. A view of generic meta-model components (Basic package)

other functions like controlling repeaters, sensors, actuators and some other server facilities.

- Concentrator is used to model a collecting place of data coming from repeaters, sensors, actuators before sending them to routers.

-Sensor_ActuatorDevice is used to capture measurement data and to send them or to act pursuant to an order.

- Repeater is used to ensure the link between a Concentrator and Sensor_ActuatorDevice elements if they are not within reach.

- RadioModule models the device that ensures exchanging data messages between all other devices when wireless communication links are used.

Since TNE devices are specialized from the generic element Devices, they inherit all properties and references.

2) Terra Nova Energy's off the shelf devices: This second set of elements represents real devices ready to use in the framework. They are another level of specialization of the first set. They may be grouped in a kind of library composed with industrial devices coming from different manufacturers and in order to be integrated into TNE systems. In this paper we neither describe this library of devices nor the manner of their built because we focus on some other properties and aspects of model analysis.

In the next section, we propose a methodology of analysis showing the usefulness of these presented meta-models, and their examination and validation on real industrial cases.

\section{MODEL ANALYSIS}

From the TNE domain specific meta-model, presented in the previous section, an instance that describes a real system of telecontrol may be defined. According to the user needs, this instance captures a particular concern for a system and gives the necessary elements and their relevant properties.

\section{A. First case study with a complete TNE's instance}

In this first case study, an instance of a TNE meta-model has been created with a tree editor It is an abstraction of a TNESystem composed of two BoxaNova that manage twenty
Sensor_ActuatorDevices. Each BoxaNova consists of two Concentrator elements and a Router. Communication between these components uses two Mediums; a coaxial cable that connects the first concentrator and the router and a RS232 cable that provides serial communication between the second concentrator and the router.

Sensor and actuator devices use a ZigBee protocol[11] to communicate with the BoxaNova concentrators. To achieve this task, they use radioModules. Concentrators also have their own radio modules. In general, wireless propagation environments in TNE systems may have different attenuations. Thus, each communication between a sensor/actuator and its repeater or concentrator is defined by a medium with relevant properties and necessary (contact) interfaces. This information is kept by properties such as the attenuation value, speed of transmission, signal length, etc.

The model also captures other information like the physical location ( $\mathrm{x}, \mathrm{y}$ coordinates) of the different parts of the system. In this way, Communication vs. placement analysis have been conducted. In fact, component's placement in TNE sytems is crucial to ensure good communication and proper information transfer. As the model is fully known, an analyzer can be created to verify that every Sensor_ActuatorDevice element according to its coordinates may communicate to its corresponding concentrator. From information analysis text file obtained (see transformation model to text in [7]), manual corrections may be realized.

This task is manageable for small systems but gets difficult and very expensive for large ones with many components. The next part gives an illustration of a methodology that can treat this difficulty.

\section{B. Second case study with a partial TNE's instance}

In this second case study, only the number and the position of sensor/actuator devices are given. The instance is partial, and the objective is to automatically find a solution for repeater's and concentrator's placement. This problem is a very complex one and several solutions may be found in literature[12][11][13].

In our case an input model is generated automatically with 
the required properties. As a first step, an instance of the TNE meta-model is created with a system TNESystem, a number of sensor_ActuatorDevice and an environment that represents a factory hall. We suppose that radio measurements were made in the environment and have identified attenuation values.

In a second step, an analysis engine for placement, composed of three analysers, is used:

1) The primary analyzer performs an initial refinement of the input model. First, it divides the environment, specified in the input model, into zones where mediums have the same attenuation Then, depending on the number of sensor/actuator devices, it creates the necessary repeaters with their radio modules, and adds them to the input model with coordinates in the vicinity of the centre of the sub-environment. Finally, it creates connections (ContactInterface) between repeaters and sensor/actuator elements and adds them to the model.

2) A second pass of refinement with the same treatment approach is made by the second analyzer. Its objective is to connect repeaters with concentrators. The input model environment is split up into several zones with new radio modules ranges of repeaters. The number of added concentrators will depend on their capacity and also on the number of repeaters within reach.

3) The third analyzer performs an optimization on the obtained model that contains sensor/actuator devices that can communicate directly with concentrators without the use of a repeater.

Figure 2 shows a simple placement layout of TNE system components obtained with the following initial model inputs: 200 sensors/actuator devices, an environment that represents a factory with 800 meters length and 600 meters width and an attenuation value of 5 decibels by meter. Red rectangles represent repeaters, blue triangles represent concentrators and green circles represent sensor/actuator devices.

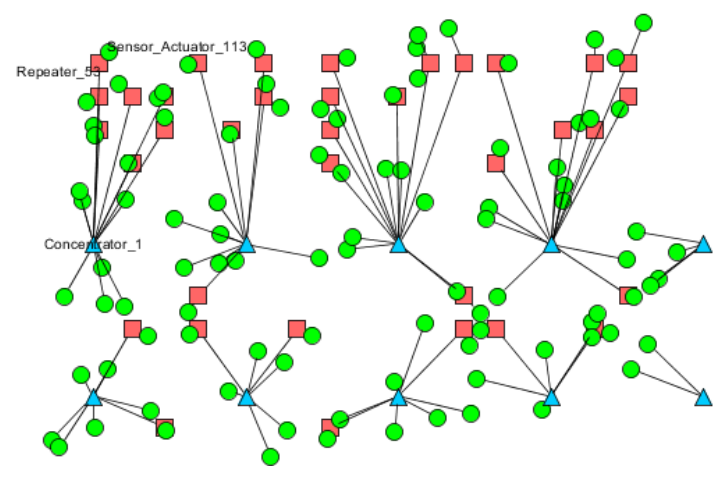

Fig. 2. Placement layout (partial view)

The generated model allows us to have a proposal for the positions of the repeaters and of the concentrators in the environment. This obtained placement is not optimal, as simple hypothesis have been used. Nevertheless, our objective is to show that using model analysis in ubiquitous systems in MDE approach may support engineers in the design of their systems and verifying some properties in earliest stage without real implementation such as communicating object placement.

\section{CONCLUSION AND FUTURE WORKS}

In this paper a generic meta-model for modeling ubiquitous telecontrol systems and, specially telecontrol systems is proposed. A variant of this metamodel is specified to fit the needs of Terra Nova Energy.

The introduction of the Model Driven Engineering approach in this field allows us to exceed the contemplative dimension of models towards productive models. The presented cases studies highlights this approach by proposing a possible model for placement analysis, using transformations.

In the future, we would like to improve our generic metamodel in order to take the behaviour of the different components into account. We would like to move from static analysis to simulation with integration of a system inside its environment.

\section{ACKNOWLEDGMENT}

This work is supported by Brest Métropole Océane, and performed in partnership with Terra-Nova Energy.

\section{REFERENCES}

[1] M. Weiser, "The computer for the 21st century," Scientific American Special Issue on Communications, Computers, and Networks, 1991.

[2] P. Le Parc, A. Touil, and J. Vareille, "A model-driven approach for building ubiquitous applications," in The Third International Conference on Mobile Ubiquitous Computing, Systems, Services and Technologies - UBICOMM 2009, Oct. 2009.

[3] S. Kent, "Model driven engineering," Lecture notes in computer science, pp. 286-298, 2002.

[4] F. Fleurey, J. Steel, and B. Baudry, "Validation in model-driven engineering: testing model transformations," in Workshop WS5 at the 7th International Conference on the UML, Lisbon, Portugal, 2004.

[5] S. Gerard, F. Terrier, and Y. Tanguy, "Using the model paradigm for real-time systems development: Accord/uml," Lecture notes in computer science, vol. 2426, pp. 260 - 269, 2002.

[6] S. Cranefield and M. Purvis, "UML as an ontology modelling language," in Proceedings of the Workshop on Intelligent Information Integration, 16th International Joint Conference on Artificial Intelligence (IJCAI-99), vol. 212, 1999.

[7] K. Czarnecki and S. Helsen, "Classification of model transformation approaches," in Proceedings of the 2nd OOPSLA Workshop on Generative Techniques in the Context of the Model Driven Architecture, 2003.

[8] T. Alenljung and B. Lennartson, "Formal Verification of PLC Controlled Systems Using Sensor Graphs," in Proceedings of the fifth annual IEEE international conference on Automation science and engineering. The Institute of Electrical and Electronics Engineers Inc., 2009, pp. 164-170.

[9] A. Voronov and K. AAkesson, "Verification of process operations using model checking," in CASE'09: Proceedings of the fifth annual IEEE international conference on Automation science and engineering. The Institute of Electrical and Electronics Engineers Inc., 2009, pp. 415-420.

[10] I. C. Committee, "A consensus of the incose fellows," International Council On Systems Engineering, Tech. Rep., 2006. [Online]. Available: http://www.incose.org/practice/fellowsconsensus.aspx

[11] P. Baronti, P. Pillai, V. Chook, S. Chessa, A. Gotta, and Y. Hu, "Wireless sensor networks: A survey on the state of the art and the 802.15. 4 and ZigBee standards," Computer Communications, vol. 30, no. 7, pp. 16551695, 2007.

[12] S. Ghosh and S. Rao, "Sensor network design for smart highways," in CASE'09: Proceedings of the fifth annual IEEE international conference on Automation science and engineering. The Institute of Electrical and Electronics Engineers Inc., 2009, pp. 353-360.

[13] X. Cheng, D. Du, L. Wang, and B. Xu, "Relay sensor placement in wireless sensor networks," Wireless Networks, vol. 14, no. 3, pp. 347$355,2008$. 